\title{
THE CHEMOTHERAPY OF TROPICAL INFECTIONS OF THE BOWEL
}

\author{
By A. R. D. Adams, M.D., F.R.C.P., D.T.M. \\ Physician for Tropical Diseases to the Royal Liverpool United Hospital, Sefton General Hospital and to the \\ Childwall Hospital, Ministry of Pensions, Liverpool
}

Pathogenic infections of the bowel are peculiarly prevalent in the tropics, where sanitary habits are primitive and flies and other vectors abound. It is incorrect to regard many of these infections as peculiar to the tropics; indeed most of them also are found in the temperate and the cold parts of the globe: The bacterial infections include those responsible for the bacillary dysenteries, the enteric fevers and cholera; the protozoal infections include those which lead to amoebic dysentery, and to the diarrhoeas which have been ascribed to flagellate or to ciliate protozoad parasites; and there are a number of intestinal helminthic infections which may or may not give rise to clinical manifestations. It is with the present position of chemotherapy in the treatment of some of these that this article is concerned.

\section{The Bacterial Infections}

\section{The Bacillary Dysenteries}

Until shortly before the recent war there was no specific drug treatment for the bacillary dysenteric infections. American workers about that time observed that sulphanilyl guanidine (sulphaguanidine), when given orally to experimental animals, was not absorbed to a material extent from the intestine, and that the bacterial flora of the stools of animals treated with it were markedly modified. They therefore suggested that this drug might prove to be of therapeutic value in those bacterial diseases of man in which the infection was confined to the lumen of the intestine. There are two outstanding examples of this; cholera, in which the infection is located in the small bowel, and the bacillary dysenteries, in which it is located in the large bowel. Marshall et al. (194I) treated a number of children suffering from proven bacillary dysenteric infections in the United States with sulphaguanidine. The results, when the children were treated in the first week or so of the disease, were dramatic, the children becoming convalescent within two or three days. The dosage advocated by these workers was an initial one of 0.1 to $0.2 \mathrm{gm}$. per $\mathrm{kgm}$. body weight, followed by $0.05 \mathrm{gm}$. per $\mathrm{kgm}$. four-hourly until the number of stools was not more than four in 24 hours; the drug was then continued at eighthour intervals in similar dosage for a further three days into convalescence.

This fundamental observation obviously was of the greatest importance in connection with the welfare of troops engaged in warfare overseas, especially those in the Mediterranean area where bacillary dysentery had wrought such havoc in the preceding world war. Fairley and Boyd (1943) treated men in the Forces suffering from acute attacks of bacillary dysentery in these ? regions and obtained equally satisfactory results; it was evident that the problem of the specific treatment of patients suffering from the bacillary dysenteries was solved. As sulphaguanidine was at first not readily available in all areas of military activity, other sulphonamide preparations were at times used; these, most of which were readily absorbed from the intestine and so did not reach the lumen of the large bowel directly, also proved effective in the treatment of acute bacillary dysentery. Their use in some cases was attended by undesirable side effects, in particular renal obstruction due to crystalluria; the tendency to this increased when compounds prone to cause it were given to patients suffering from a dehydrating febrile disease, such as this, in a hot climate. Some deaths from renal obstruction would have occurred in at least one reported instance, from the employment of sulphapyridine, had not facilities for renal lavage been readily available. The employment of the freely-absorbable sulphonamides in the treatment of bacillary dysentery in British forces was discouraged on this account, and British workers still prefer to use the poorlyabsorbed preparations in this condition.

Following the employment of sulphaguanidine on an enormous scale among the Forces, two 
further sulphonamide preparations, with the similar characteristic of limited absorbability from the intestine, were developed. These were succinyl sulphathiazole (sulphasuccidine) and phthalyl sulphathiazole (sulphathalidine); both were stated to be more effectively bacteriostatic than sulphaguanidine. Sulphasuccidine is usually now that selected as the most efficient of these three compounds by British workers for the treatment of the bacillary dysenteries.

American authorities are less averse to using the readily-absorbable sulphonamides. Mackie et al. (1945) list, in order of effectiveness, sulphadiazine, sulphathiazóle, sulphapyridine, sulphasuccidine and sulphaguanidine for the treatment of the bacillary dysenteries. They emphasize the importance of the maintenance of an alkaline urine during sulphonamide treatment, to minimize the risk of renal complications, and advocate the administration of 30 to $60 \mathrm{gr}$. of sodium bicarbonate with each dose of the drug employed, to this end. Hardy (1946), however, places the sulphonamides in the following order of efficacy for the treatment of cases of bacillary dysentery: sulphapyrazine, sulphadiazine, sulphasuccidine, sulphamerazine, sulphamethazine, sulphathiazole, sulphaguanidine, sulphapyridine and sulphanilamide. He stresses the need for restoring and maintaining a normal state of hydration before giving these compounds.

There is therefore agreement by these workers that sulphadiazine is among the most effective of the sulphonamides in the treatment of the bacillary dysenteries, but some disagreement on the order of merit in which the other compounds can be placed. But it is rather interesting to note that in cases proving resistant to a week's treatment with sulphadiazine or sulphathiazole, sulphasuccidine has been stated to be of great value. In clinical practice, both sulphadiazine ( 4 to $6 \mathrm{gm}$. daily) of the freely-absorbable compounds, and sulphasuccidine ( $20 \mathrm{gm}$. daily) of the poorly-absorbable compounds, give closely approximating results. There would thus seem to be no advantage in departing from, and much to commend, the current British practice of using the latter in preference to the former, particularly under field conditions. In those patients in whom a particular compound is badly tolerated or produces undesirable sideeffects, for example vomiting, another may be employed, with suitable modification in dosage. While infections with Flexner and Shiga types of dysentery bacilli usually prove readily amenable to one or other of these drugs, the much less virulent Shigella sonnei infections do not always do so.

While at first it was considered that sulphaguanidine was not absorbed to any material extent from the intestine, further experience has led to some modification of this view. It appears, for气̊ instance, that in cases of old-standing and ex- $\frac{3}{\infty}$ tensive ulceration of the large intestine sulpha- $\stackrel{\varnothing}{\circ}$ guanidine, and the allied compounds, may be much $\complement$ more freely absorbed than was originally thought $\overrightarrow{\vec{F}}$ possible. Their administration in excessive or un- $\frac{-}{0}$ duly prolonged dosage, particularly to such cases, $\frac{\bar{\sigma}}{\sigma}$ is therefore to be avoided.

The question of drug resistance inevitably arises $\frac{\pi}{\widehat{D}}$ in considering the chemotherapy of bacillary in- $\frac{\Omega}{0}$ fections. There is little evidence that drug-fast $\odot$ strains of the dysentery bacilli were encountered $\overrightarrow{0}$ among the large numbers of cases treated with $\overrightarrow{-}$ these compounds during the war years. Since $\mathscr{\sigma}$ then Hardy (1946) has recorded the developmento of sulphonamide resistance by a strain of Flexner 3 $\mathrm{Z}$, which was found to be the cause of an outbreak of bacillary dysentery in mentally defective. children in an institution. Initially sensitive to $\omega$ sulphadiazine, after inadequate treatment with $\vec{\Delta}$ the drug and passage to fresh patients this strain ultimately became completely refractory to it, ando the clinical course of the disease thereafter was not ${ }_{-}$ affected by its administration.

Hardy (1948) treated 37 children suffering from bacillary dysentery with streptomycin given orally. $\overrightarrow{\mathbb{D}}$ The numbers of dysentery bacilli in their sto is rapidly diminished and all stool specimens wege free from them in six days, but the organisms appeared in the stools a week later in about haf the cases. This antibiotic, which is poofy absorbed from the gut when given orally, ap-o parently acted locally; in these circumstances it did not cause the toxic manifestations sometimeso associated with this compound. Streptomycin命 was markedly inferior in therapeutic action to sulphadiazine, which was concurrently used ino the treatment of other cases in the same institution. No evidence was forthcoming of the development of streptomycin resistance by the organisms in the cases treated with this drug, some of which, it is stated, were already sulphonamide resistant as judged both by clinical and by in vitro studies. It may be that streptomycin will find some placei in the treatment of bacillary dysentery in cases of infection with sulphonamide resistant strains of bacilli.

\section{The Enteric Fevers}

The typhoid infections of course are not corn fined to the bowel, but are systemic infections. Nevertheless, in view of the extensive lesions which occur in the intestine in these fevers, and the im portance of these in the symptomatology, it is justifiable to consider the typhoids when dis $\frac{\pi}{\mathbb{D}}$ cussing infections of the bowel.

On the introduction of the sulphonamides their use in the treatment of the typhoid fevers soon re- 
ceived attention. The results were not encouraging, and it was evident that though the sulphonamides might play a part in the control of the secondary bacterial infections of the lesions in the bowel they did not exert any effective specific action on the causative organism. Bigger (1946) demonstrated that cultures of Bact. typhosum in broth freed from sulphonamide-antagonizing substances were inhibited by the addition of sulphathiazole; he showed that penicillin in high concentrations reduced the growth in similar cultures, and he finally demonstrated that a combination of sulphathiazole and of penicillin exerted a pronounced bactericidal effect on the cultures. He suggested that a combined penicillin and sulphathiazole treatment, which he outlined, might produce serum concentrations of the drugs in man which had been found effective in vitro, and that this treatment was worthy of trial in cases of typhoid fever.

His suggestion was implemented by McSweeney (1946) who treated in Ireland six cases of proven Bact. typhosum infection in this manner, and who stated that the clinical results confirmed Bigger's expectations. Though some of these patients suffered relapses and required a further course of treatment the immediate results were considered by McSweeney to be most encouraging. As a consequence combined sulphonamide and penicillin treatment was widely used, but soon the consensus of opinion was that the treatment was not effective.

The effective chemotherapy of the enteric fevers therefore remained an unsolved problem until 1948, when Smadel (1950) and his associates, while investigating the treatment with chloramphenicol (chloromycetin) of cases of mite-borne typhus, employed this drug on two patients subsequently proved to be suffering from typhoid and not from typhus. The response of these to the treatment was unexpectedly favourable, and detailed studies of the effect of chloramphenicol in typhoid were accordingly begun. Forty-five patients with typhoid were treated with the drug; only one of these died from the disease, a mortality very considerably less than that in control cases. Other workers in all parts of the world have since confirmed that chloramphenicol acts specifically in arresting acute attacks of the typhoid fevers. The temperature falls within four days and the clinical improvement thereafter is striking; it has been established that the bacteraemia ends appreciably before either the temperature or toxaemia disappear.

Smadel and his associates in their early work in Malaya gave an initial dose of $3 \mathrm{gm}$. of chloramphenicol by mouth for the treatment of typhoid, and this was followed by $3 \mathrm{gm}$. daily until the temperature became normal; thereafter they gave I or $2 \mathrm{gm}$. daily for variable periods. They found that when the drug was discontinued within eight days there was a high relapse rate of the disease, but when chloramphenicol treatment was continued for 14 days or more there were few relapses. Others have had similar experiences, and it is evident that the drug must be continued over an adequate period, either continuously or, as suggested by some of the American workers, after an intermission of five days, until at least the third week or relapse is prone to occur.

The action of chloramphenicol on the infection is merely bacteriostatic, and its actual eradication is the result of immune body formation by the patient. In vitro experiments support this view as, though chloramphenicol inhibits growth in cultures of Bact. typhosum, it does not kill the bacilli; furthermore, chloramphenicol does not sterilize chronic carriers of this organism of their infections; in this it falls short of the complete specific.

There have been no reports of the development of drug-fastness to chloramphenicol by strains of Bact. typhosum. In one case of typhoid personally seen, which was treated intermittently for short periods with chloramphenicol as relapses recurred over three months, no clinical evidence of the development of resistance to the drug by the infecting strain was observed. Each relapse re-co sponded as regularly to retreatment as did the ? initial attack.

The antibiotic aureomycin has been used in the treatment of typhoid, but initial reports show it to act much less effectively than does chloramphenicol in this disease. Streptomycin and polymixin, two potentially toxic antibiotics, also show some evidence of action in enteric. None of these compete with chloramphenicol, at present the drug of choice in the specific treatment of the enteric fevers.

A further advance in the chemotherapy of typhoid is reported in a recent paper by Smadel and his colleagues (195I). Increase in the amount of adrenal cortical hormones has been shown to exert a beneficial action on the clinical course of a number of infectious diseases. The clinical improvement in cases of typhoid treated with chloramphenicol is delayed, often for some days, after the start of treatment with this drug. Cortisone acetate was given concurrently with chloramphenicol, during the first few days of treatment with the latter, to a number of cases of typhoid. This was found markedly to expedite the clinical response to treatment. A dosage of 5 to $6 \mathrm{mgm}$. per $\mathrm{kgm}$. body-weight of cortisone on the first day, followed by decreasing amounts for another few days, resulted in the disappearance of 
Special lectures by neurologists from outside London and from abroad are held throughout the three courses. Fees, for the ten weeks' full-time course 20 guineas; for attending hospital practice, 15 guineas for three months or 28 guineas for six months.

Part-time teaching is given in the Out-patient Department, at the National Hospital, Queen Square, on five days a week throughout the year (public holidays excepted), and at Maida Vale Hospital.

Courses of Clinical Demonstrations are given on Wednesdays and Saturdays at the National Hospital, Queen Square. Advertisements concerning these appear from time to time in the medical journals.

For further particulars please apply to the Dean, Institute of Neurology, National Hospital, Queen Square, London, W.C.1.

\section{INSTITUTE OF OBSTETRICS AND GYNAECOLOGY (British Postgraduate Medical Federation)}

Incorporates the teaching facilities of Queen Charlotte's Maternity Hospital, the Chelsea Hospital for Women, and the Department of Obstetrics and Gynaecology of the Postgraduate Medical School. Graduates must hold a registrable medical qualification.

On enrolment, graduates are posted to one of the constituent hospitals and also attend combined classes at the other two hospitals. The enrolment fee is $£ 3$, and the charge for one term of 12 weeks is $£ 3^{\circ}$.

An intensive course suitable for those preparing for higher examinations will be held in December and June.

General practitioners requiring further experience in obstetrics are accepted during the term at Queen Charlotte's Hospital, to attend the practice of the hospital for two or four weeks, with opportunity to conduct normal deliveries and to attend combined classes at the other hospitals. Fee $£_{3}$ a week during term. Ministry of Health grants are payable to approved general practitioners attending for such a period. General Practitioner Refresher Courses, lasting one week, are həld twice a year for which the fee is $£ .55$ s. This course is recognized by the Ministry of Health, in the case of approved practitioners, for the purpose of a grant.

Postgraduates may attend the practice of the hospital at the Postgraduate Medical School and Queen Charlotte's Hospital during the vacation, when a charge of $f_{1}$ a week is made.

Hostel accommodation is available at the Postgraduate Medical School and at Queen Charlotte's Hospital.

Applications for further information and for enrolment forms should be made to the Secretary, Institute of Obstetrics and Gynaecology, Chelsea Hospital for Women, Dovehouse Street, S.W.3.

\section{INSTITUTE OF OPHTHALMOLOGY}

(Royal London Ophthalmic, Royal Westminster Ophthalmic, Central London Ophthalmic Hospitals) (British Postgraduate Medical Federation)

Courses of lectures a $-d$ classes beginning March I and October I each year to meet the requirements of candidates entering for the examination for the Diploma in Ophthalmology and other ophthalmological examinations will be given by members of the staff of the Hospital and Institute. Each course is designed to extend over two terms of approximately 18 weeks each and is normallv divided into two parts.
Part I. Anatomy (including embryology and norkal histology), elementary physiology of the eye, opacs (elementary and physiological), practical tutorials refraction work and clinical subjects.

Part II. All clinical branches of the subject toget with bacteriology and pathology. Students can attend the daily clinical practice of the two branches of the Moorfields, Westminster and Central Eye Hosp丞l, and hold the appointments of Junior Clinical Assistants and Clinical Assistants concurrently with the abgye courses. A composition fee of $£_{2} 6$ s. Od. will adpit students once to the lectures and tutorial classes of any one term, with six months' clinical practice in Bhe hospital.

(1)

Extra Courses. Slit lamp microscopy (fee $£_{5} 5 \mathrm{~s}$. od.); orthoptic training (one week's intensive course, . Pee f5 5s. od.); contact lenses (one weeks' intensive course, fee $f$ ro ros. od.). Additional courses by arrangemgat. Hospital Practice only. Fees as follows: one moghh, $£ 2$ 2s. od.; three months, $£ 5$ 5s. od.; six monthis, fro ros. od.

Facilities for research.

For further information apply to the Dean, Institute $\omega_{-}^{\omega}$ Ophthalmology, fudd Street, London, W.C.1.

\section{INSTITUTE OF ORTHOPAEDICS}

\section{(British Postgraduate Medical Federation) $\vec{\infty}$}

The Institute is concerned with postgraduate educaflon in orthopaedics and with research. The practice of The $^{\text {he }}$ Royal National Orthopaedic Hospital (both at the fown section in Great Portland Street, and at the courifry section at Stanmore) is open to postgraduates, who may join at any time. In addition to hospital practice, real courses of various lengths are held during academic terms. Facilities for advanced clinical work are avalible for selected candidates having a suitable scientificoor surgical training.

Further particulars may be obtained from the D审n, Institute of Orthopaedics, Royal National Orthopafic Hospital, 234 Great Portland Street, W.1.

\section{INSTITUTE OF PSYCHIATRY}

\section{(British Postgraduate Medical Federation}

The Institute, which was formerly the Maudstiey Hospital Medical School, is now associated with the oint Bethlem Royal Hospital and Maudsley Hospital. It is concerned with postgraduate education in psychiatery and allied subjects, and with research. A course of instruction covering up to three years, and starting in October yearly, is provided for medical practitioners who wish to specialize in psychiatry or to prepare for the Diploma in Psychological Medicine.

Clinical instruction, which includes case conferenê: seminars, and practical experience in case taking ins given at associated hospitals in the mornings, and systematic lectures and demonstrations are arranged for the afternoons. Students may also enrol for shorer periods, or for single series of lectures.

The subjects covered include general and child prychiatry, principles of psychotherapy, forensic psychiąy, criminology, neuroanatomy, neurophysiology, wh special reference to the clinical applications of the electroencephalogram, neuropathology, biochemistry, sockal psychology, mental testing, and statistics. Lectures on subjects of special interest are arranged from time time. The tuition fee for a full year's courseథึis $£ 53$ ros. od., including enrolment fee. For shorter periods the fee varies with the tvpe of course chosen. 
medicine in the $17^{\text {th }}$ century for the same purpose. It was employed empirically in all forms of dysentery until the early years of this century, when the causative parasite of amoebic dysentery was discovered and the specific therapeutic effect in amoebic dysentery of its principal alkaloid, emetine, was established. Since then emetine has remained the almost universally accepted specific for the arrest of acute amoebic dysentery. Conessine, an alkaloid derived from kurchi bark, possesses similar properties, but it is gerlerally regarded as a more toxic and inferior alternative to emetine; it is not now used by the English workers although there has been a recent rise in its favour in France.

Emetine hydrochloride, given intramuscularly or subcutaneously in doses of $\mathbf{I}$ gr. daily for several days, will promptly arrest an acute attack of amoebic dysentery. It will not sterilize the infection in the bowel in more than a small proportion of cases, and no purpose is achieved by continuing the injections after the immediate therapeutic effect, arrest of the attack, is achieved. At most 12 injections should be given, as the drug is toxic and is cumulative in its toxic action; rarely is it necessary to give more than half-a-dozen injections to achieve the immediate purpose.

There is evidence that amoebae in cultures on suitable media are killed by very low concentrations of emetine. Whether such concentrations of the drug are reached in the tissues in man following the usual therapeutic treatment is open to question.

The mode of action and the fate of emetine in the body are unknown. While there is no experimental proof that resistance by the parasite to emetine is engendered, there are some grounds for thinking that repeated prolonged courses of emetine injections render a bowel infection with $E$. histolytica in man subsequently more difficult to eradicate; nevertheless, in these cases emetine does not fail to produce the immediate clinical response expected on treatment with it of a clinical relapse. It is by reason of the toxicity of the drug, and of the possibility of rendering the infection less amenable to subsequent sterilizing treatment, that unnecessary injections of it are undesirable.

(b) Complications. The complications at present under consideration are those due to extension of the amoebic infection from the large intestine. This extension may occur directly through the wall of the intestine to neighbouring structures, or by contamination and infection of tissues, such as the skin around a colostomy opening, with amoebae. It more frequently occurs by carriage of parasites in the portal circulation to the liver, with the subsequent development of an amoebic liver abscess. The latter may involve neighbouring structures by direct extension or, rarely, by metastasis may lead to the development of further amoebic abscesses in remote organs.

As in the case of an acute attack of amoebic dysentery, emetine is specific in the treatment of extraintestinal amoebic infection. More important still, and in contrast, if given in adequate dosage it sterilizes such lesions of their parasites. When, therefore, amoebic infection of any tissue is detected as a sequel to a primary gut infection a full course of 12 injections of emetine hydrochloride, I gr. daily, should at once be given. Only rarely does this require repetition to achieve the desired purpose. When the complication has been dealt with by emetine treatment, and possibly other non-specific treatment such as surgical drainage, the gut infection still remains and must be eradicated if only to forestall further extraintestinal spread of the infection.

Recently it has been observed that the new antimalarial drug, chloroquine, when given orally in therapeutic dosage is recoverable from the liver in concentrations many hundred times greater than from elsewhere. It has also been observed that this compound exerts a specifically lethal action on $E$. histolytica in the liver, and that amoebic liver abscesses are sterilized of their amoebic infections by chloroquine treatment as rapidly and as effectively as by emetine treatment. Chloroquine, therefore, is a valuable alternative to emetine in the treatment of amoebic liver abscess; it also is a most valuable substitute for emetine when the latter compound is contraindicated, as in cases with heart disease or where it causes undue evidence of toxicity. Chloroquine, however, is apparently without effect on the bowel infection and does not afford an alternative to emetine in the arrest of an acute attack of amoebic dysentery.

(c) Amoebiasis. The eradication of the residual intestinal amoebic infection after control of the acute dysenteric symptoms with emetine can be obtained by treatment empirically with a number of drugs. There is no single drug which alone gives consistently satisfactory results, so it is the usual practice to administer concurrently a number of drugs, each of which shows some activity; in practice the results are very satisfactory, though in some few cases a single course of treatment may not eradicate the infection.

The drugs commonly employed include a preparation of emetine (E.B.I.) for oral administration, an arsenical (stovarsol, carbarsone, milibis) for oral administration, and one of the iodooxyquinoline derivatives (diodoquin, chiniofon) either orally or as a retention enema. These given systematically and in concert over a period of two, or preferably three, weeks will be found to sterilize 
the great majority of amoebic bowel infections. The details of one such method of treatment, which has proved very effective, are appended.* The emetine-bismuth-iodide (E.B.I.) used in this particular course is contained in a very satisfactory enteric-coated pill; that the contents of this pill are liberated is evident by the nausea which indicates its absorption some three hours after its consumption. It is highly improbable that E.B.I. exerts its action locally within the bowel, as the emetine obviously is absorbed. Stovarsol may cause a rash in some individuals; on its appearance the drug must be withdrawn at once or an exfoliative dermatitis and other manifestations of arsenicalism may follow. Milibis is a new arsenical preparation in which the arsenic is combined with bismuth; it is said to be much less freely absorbed and so to be less toxic than stovarsol; the implication is that the arsenical, at least in part, acts locally within the lumen of the bowel. Diodoquin, which contains 63 per cent. of iodine, can only be given orally and as it is not absorbed to a material extent also presumably exerts its action locally within the bowel. Chiniofon, a similar preparation containing 26 per cent. iodine, is given in the form of a retention enema and also exerts a local action. As the latter is non-irritant and bacteriostatic and bactericidal, in addition to destroying the amoebae with which it comes into contact it may well be belpful in cleansing lesions reached by it.

Combined treatment with a range of compounds of this type therefore consists of a local attack on the parasites which lie within the lumen of the bowel, or on the surface of the lesions, by the drugs which are not absorbed from the intestine, and an attack on those within the tissues of the bowel wall by the drugs, such as emetine, which are absorbed and diffused systemically. The treatment though empirical is not irrational, and it has proved effective. Claims that either the arsenicals or the iodo-oxyquinolines given alone over a period will sterilize non-pathogenic bowel infestations with $E$. histolytica are numerous, but the percentage of successful results with such treatment almost invariably on more prolonged investigation is found to be disappointing.

It was claimed by Hargreaves (1945) that in oldstanding or unusually severe cases of relapsing amoebic dysentery treatment of the secondary

\footnotetext{
*On the odd days of a 2 I-day period: (I) Enteric coated E.B.I. (B.W.) I grain thrice daily. (2) Bismuth subnitrate I drachm thrice daily.

On the even days: (1) Rectal washout with 2 per cent. sodium bicarbonate, followed an hour later by retention enema of 4 per cent. chiniofon (to be retained at least 6 hours). (2) Stovarsol 4 grains thrice daily. (3) Bismuth subnitrate I drachm thrice daily.
}

bacillary infection of the intestinal lesions by $\mathrm{a} \cong$ course of a sulphonamide and penicillin, either $\frac{\pi}{\infty}$ prior to or concurrently with the antiamoebic $\stackrel{\varrho}{c}$ treatment, promoted the clinical recovery of the $\Rightarrow$ patient and also made the eradication of the $\stackrel{\vec{s}}{\overrightarrow{0}}$ amoebic infection more certain. Some workerso accordingly use these preparations, particularly in $\overline{0}$ the case of patients passing pus cells in the stools, $\frac{\bar{p}}{\bar{D}}$ in conjunction with a combined course of treatment $\stackrel{\square}{\Omega}$ with a variety of anti-amoebic drugs.

Within the last few years aureomycin given by the mouth, and without other treatment, has been reported to sterilize bowel infections with $E \cdot \overrightarrow{\vec{\omega}}$ histolytica. Claims of cure in from 60 per cent. to 100 per cent. of the cases treated have variously응 been made. McVay et al. (1949), who first re- 3 . ported on the effect of aureomycin on amoebiasis, $y$ suggested that its action was a direct one on the $\omega$ parasites. Fuller and Faust (1949), and others, $\overrightarrow{-}$ have shown that aureomycin in vitro is not $\vec{N}$ amoebicidal in great dilutions; concentrations $v$ greater than $1 / 100,000$ are necessary to sterilize $\frac{\text { S }}{5}$ cultures of the growths of amoebae, and their $\vec{c}$ action may be ascribable to the destruction of the $D$ bacillary growths in the cultures rather than $a \frac{\overrightarrow{+}}{+}$ directly amoebicidal effect. It has become evident 3 that some of the initial claims as to the therapeuttc ${ }_{\mathbb{\Phi}}$ efficacy of aureomycin in this condition were us duly optimistic. Of some three dozen patient of with amoebiasis or suffering from uncomplicates amoebic dysentery, treated in a local hospital in England solely with aureomycin, about two-thirds were freed of their infections. It would appear that the drug probably is not primarily amoebicidal $\cong$

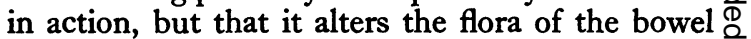
and so renders the environment unsuitable for the $\overrightarrow{\vec{P}}$ maintenance of $E$. histolytica. Nevertheless aureo- $\frac{3}{3}$ mycin is a valuable addition to the range of drugs influencing the infection. Precisely the same can be said of terramycin and of bacitracin; but streptomycin and some of the other antibiotics 3 . appear to be of little value, probably because they are effective only against types of bacteria nonessential to the welfare of the amoebae.

The evidence suggests that none of the antibiotics alone should be relied upon to free the ? bowel of $E$. histolytica infection, but that one of $D$ them may with advantage be given concurrently with the combined course of anti-amoebic treat- $\bar{N}$

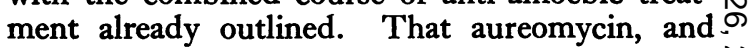
terramycin, are valuable adjuvants in such treat- $N$ ment is indicated by the results of treatment with $\underset{\omega}{N}$ them of two or three very abnormally resistant $\sigma^{2}$ cases of chronic relapsing amoebic dysentery, 0 repeatedly treated unsuccessfully with an extremely large variety of drugs in various com- $\stackrel{\oplus}{+}$ binations at intervals over a period of years and by $T$ a number of physicians. In these cases, personally 
treated, aureomycin given alone produced only temporary freedom of the stools from parasites, as indeed had the various combined courses already given. But when aureomycin was given by mouth in doses of $0.5 \mathrm{gm}$. six-hourly for the first ten days of a combined course, permanent parasitic sterilization of the bowel was ensured. This was determined by the subsequent periodical examination of a dozen daily specimens of stool at three monthly intervals over two years, and by the complete freedom of the patient during this period of observation from symptoms ascribable to a persistent infection. In a similar case terramycin produced a comparable reșult.

\section{Other Protozoal Infections}

Of some half-dozen protozoan parasites other than Entamoeba histolytica which commonly are found infesting the human bowel, only the flagellate Giardia lamblia can be regarded as a possible pathogen. This parasite, which adheres to the surface of the mucosa chiefly of the uppermost portion of the small intestine, is believed by some to cause simple diarrhoea, or in some cases of unusually heavy infestations, dysenteric diarrhoea. A course of treatment with mepacrine hydrochloride by mouth, in doses of 0.1 to 0.2 gm. thrice daily for five days, almost invariably eliminates the infection.

\section{BIBLIOGRAPHY}

BHATNAGAR, S. S., DE SA, J., FERNANDES, F., and DIVEKAR, P. V. (1948), B.M.H., I, 719 .

BIGGER, J. W. (1946), Lancet, i, 81.

FAIRLEY, N. H., and BOYD, J. S. K. (1943), Trans. Roy. Soc. Trop. Med. and Hyg., 36, 253.

FULLER, F. W., and FAUST, E. C. (1949), Science, $110,509$. HARDY, A. V. (1946), U.S.A. Pub. Hlth. Repts., Washington, 6r,

HARDY, A. V., and HALBERT, S. P. (1948), Ibid., 63, 790.

HARGREAVES, W. H. (1945), Contribution to symposium, Ann. Roy. Soc. Trop. Med. and Hyg., 38, 237.

LAHIRI, S. C. (1951), Lancet, i, 500.
MACKIE, T. T., HUNTER, G. W., and WORTH, C. B. (1945), 'A Manual of Tropical Medicine,' W. B. Saunders Co., Philadelphia and London.

MCSWEENEY, C. J. (1946), Lancet, ii, II4.

MCVAY, L. V., LAIRD, R. L., and SPRUNT, D. H. (1949), Science, 109, 590.

MARSHALL, E. K., BRATTON, A. C., EDWARDS, L. B., and WALKER, E. (1941), Bull.' Fohns Hopk. Hosp., 68, 94.

SMADEL, J. E. (1950), Trans. Roy. Soc. Trop. Med. and Hyg., 43, 555.

SMADEL, J. E., LEY, H. L., and DIERCKS, F. H. (i95I), Ann Int. Med., 34, I. 